\title{
Hybrid-Training Method Increases Muscle Strength and Mass in the Forearm without Adverse Effect of Hand Function in Healthy Male Subjects
}

\author{
HIROO MATSUSE, CHIKAHIRO IWASA, KISEI IMAISHI, TAKESHI NAGO, \\ YOSHIHIKO TAGAWA*, TATSUYUKI KAKUMA** AND NAOTO SHIBA \\ Rehabilitation Center, Kurume University Medical Center, Kurume 839-0863, *Department of Mechanical and \\ Control Engineering, Kyushu Institute of Technology, Kitakyushu 804-8550 and \\ **Biostatistics Center, Kurume University, Kurume 830-0011, Japan
}

Received 28 July 2010, accepted 29 October 2010

Edited by NAOKO YOSHIDA

\begin{abstract}
Summary: Conventional neuromuscular electrical stimulation (NMES) results in surface muscle contraction but high electrical stimulation intensity is required to activate the deep muscles. Therefore, NMES is not useful for training at complicated sites such as the forearm. To make NMES more effective we developed a hybrid training method (HYB), consisting of electrically stimulated antagonists to resist agonist muscle contractions. The purpose of this study was to compare the effects of HYB on the forearm as compared with NMES alone, and to determine whether HYB had any adverse effects on complex hand movements. Thirty subjects were randomly distributed into three groups: a HYB program group, an isometric electrical stimulation group (ES), and a control group (CN). Subjects trained 3 times a week for 6 weeks. Each session consisted of 10 sets of 10 reciprocal 2 -sec wrist flexions and extensions separated by 1-min rest intervals. Wrist flexionlextension torques, grip strengths (GS), forearm flexorlextensor cross sectional areas (CSA), and hand dexterity (Purdue Pegboard (PEG) test, finger tapping (Tapping) test were measured. The HYB group demonstrated statistically significant increases in wrist extension torques $(22.8 \%, \mathrm{p}<0.01)$, forearm flexor CSA $(9.6 \%, \mathrm{p}<0.01)$, and in forearm extensor CSA $(5.1 \%$, $\mathrm{p}<0.05$ ) at the end of training. There was no increase in torque or CSA in the ES or CN groups. Hand dexterity showed no significant differences in any of the three groups. HYB had no adverse effect on hand function and was more effective in forearm training than NMES alone.
\end{abstract}

Key words electrical stimulation, training, hand function, muscle strength

\section{INTRODUCTION}

Neuromuscular electrical stimulation (NMES) is widely used to lessen immobilization-associated muscle atrophy, to strengthen muscles, and to improve function in people with neuromuscular disabilities [1-5]. However, NMES has some limitations. For example, Henneman's size principle [6], (which states that in electrical stimulation activation occurs sequentially from Type I to Type II fibers according to the training intensity seen in voluntary muscle contraction) is not seen in electrical stimulation [7]. NMES activation is nonselective with regard to the type of motor unit and synchrony, unlike voluntary contrac-

Corresponding author: Hiroo Matsuse MD PhD, Rehabilitation Center, Kurume University Medical Center, 155-1 Kokubu machi, Kurume 839-0863, Japan. Tel: 81-942-31-7568 Fax: 81-942-35-0709 E-mail: matsuse_hiroh@kurume-u.ac.jp

Abbreviations: Assembly, assembly test; BH, both hands; CN, control group; CSA, cross sectional areas; ES, electrical stimulation group; GS, grip strengths; HYB, hybrid training method; LH, left hand; MRI, magnetic resonance imaging; MVC, maximum voluntary cntraction; NMES, neuromuscular electrical stimulation; PEG, Purdue Pegboard; RLB, right plus left plus both hands; RH, right hand; ROM, range of motion; SD, standard deviation; Tapping, finger tapping; VC, voluntary muscular contractions. 
tion $[7,8]$. Moreover NMES only stimulates the muscle on which the electrodes are placed, whereas voluntary movement implies activation from several synergic and stabilizer muscles, which are not stimulated by NMES [9]. These nonphysiologic patterns of activation may lead to less effective strengthening and may also contribute to the reluctance of many physicians to accept electric stimulation as a common component of therapeutic muscle strengthening programs. Also, it is generally reported that NMES evoked using surface electrodes results mainly in surface muscle contractions, and that it is hard to activate the deep muscles with this method [8,9]. Moreover NMES requires a high stimulation intensity to obtain an adequate muscle strengthening effect [8]. However high intensity NMES causes discomfort when used at sites with many superficial peripheral nerves or where cutaneous sensation is sensitive. Therefore stimulation intensity has been limited by subjects' tolerance [10]. The forearm is one of the sites where high stimulation intensity is problematic. A NMES method is needed that can provide an adequate muscular strengthening effect even at a comfortable, low stimulation intensities.

To make up for this limitation of NMES, a combined application of NMES and voluntary muscular contractions (VC) has been found effective and optimal in rehabilitation for immobilization or surgery $[8,9]$. Another new combined technique, the hybrid training method (HYB), has recently been developed (US patent 6,456,885.2002) [11]. This method resists the motion of $\mathrm{VC}$ agonist muscle with a force generated by an electrically stimulated antagonist. HYB was tested successfully for improving muscle strength and mass in healthy subjects [11-13]. Takano et al. [14] showed that HYB with low stimulation intensity increased strength and mass of the thigh muscles in elderly subjects.

An approach that can be used everywhere on the body is needed, because muscle weakness can occur anywhere. For example, in rheumatoid arthritis or aging, muscle weakness may occur in the upper extremities $[15,16]$. Especially in the hand and the forearm, not only muscle strength but also hand function decreases, so the effect of these training approaches on hand function must be considered [15]. High intensity exercise generally has a negative effect on hand function [16]. On the other hand, NMES does not facilitate intermuscular coordination [9]. Indeed, Vaz et al. [17] showed that NMES on wrist with hemiplegic cerebral palsy increased wrist strength without changing hand function. Moreover in a stroke patient's paretic (spas- tic) hand, NMES of Ia fibre in the extensor carpi radialis muscle is thought to induce reciprocal Ia-inhibition in the hand and finger flexors [18]. However, the effect of a combined application of NMES and VC on hand function and the specific coordination of complex movements is still unclear $[8,9]$. Therefore, it is necessary to examine whether HYB has an adverse effect on hand function when improving the muscle strength and hypertrophy in the forearm.

The present study was designed to compare the effect of HYB with NMES alone on the forearm in healthy subjects and to assess whether HYB for the forearm has an adverse influence on hand function.

\section{MATERIALS AND METHODS}

\section{Participants}

The Ethics Committee of Kurume University and the Japan Aerospace Exploration Agency approved the clinical design of this study protocol. Following approval, informed consent was obtained from a convenience sample of 30 healthy self-identified right hand dominant sedentary men (age, 21.4 \pm 2.5 ( \pm standard deviation (SD)) yr; height, $171.6 \pm 4.8 \mathrm{~cm}$; and weight, $63.4 \pm 8.4 \mathrm{~kg}$ ) who had reviewed the goals of the study and agreed to participate. Inclusion criteria for the subjects were as follows; unremarkable musculoskeletal conditions, no adverse medical history, no excessive alcohol consumption and/or smoking, right hand (RH) dominant according to the Edinburgh Inventory [19], and having avoided participation in any strenuous or upper extremity specific sports activities for at least a year. Subjects meeting the above criteria were then randomized into three groups matched in age, in weight, and in height: the HYB Program Group (HYB, $\mathrm{n}=10$ ) participated in a combined electrical stimulation-volitional contraction exercise program, the Electrical Stimulation Group (ES, $n=10$ ) took part in a conventional (isometric) NMES program, and the Control Group $(\mathrm{CN}, \mathrm{n}=10)$ had no specific exercise program.

\section{Intervention}

Subjects attended a single familiarization session one month prior to beginning a 6-week 3-times-aweek (Monday, Wednesday, and Friday) training program. Each exercise session consisted of the participant being seated and voluntary or electrically performing 10 sets of 10 reciprocal wrist flexion and extension contractions with their non-dominant left upper extremity. Contractions were $2 \mathrm{sec}$ in duration, and sets were separated by 1-min rest intervals. An 
exercise session required $15 \mathrm{~min}$ and $40 \mathrm{sec}$ to complete.

HYB Group: Exercises were performed in the seated position with the subject's radial and ulnar carpal flexor stimulated as the subject volitionally extended the wrist while opening the hand; next the reverse occurred (i.e., the radial and ulnar carpal extensor was stimulated) as the subject volitionally flexed the wrist while closing the hand. The range of motion of the joint was measured using a goniometer, and was restricted to an arc from a $45^{\circ}$ wrist extension to a $45^{\circ}$ wrist flexion. The subject changed the direction of the muscle contractions whenever they heard a tone emitted by the stimulator.

ES Group: These subjects were seated in the same posture as the HYB group described above. The procedures and number of sessions were identical to the HYB group with the exception that the subjects were instructed to avoid volitional contraction of the muscle; furthermore the left wrist was immobilized by a brace at the neutral position.

CN Group: Subjects did not exercise at all during this experiment period.

Equipment: The HYB apparatus consisted of an electrical stimulator, surface electrodes, and a joint motion sensor that triggered stimulation of the antagonist once it sensed the initiation of a volitional contraction [12]. Low impedance gel-coated electrodes (Sekisui plastics. Co., Ltd., Tokyo, Japan) placed over the motor points of the radial/ulnar carpal flexor and extensor have been previously described [11-14].

Waveform: Stimulation parameters were based on a standard Russian waveform in which a $5 \mathrm{kHz}$ carrier frequency was modulated at $20 \mathrm{~Hz}(2.4 \mathrm{~ms}$ on, $47.6 \mathrm{~ms}$ off) to deliver rectangular biphasic wave pulses [20].

Intensity: Stimulation intensities were set at the value of the threshold intensity plus two-thirds the difference between the maximum comfortable intensity and the threshold intensity [13], with the isometric contraction force limited to $25-30 \%$ of the maximum voluntary contraction (MVC). Output power was $<10$ $\mathrm{W}$, the current intensity was $<10 \mathrm{~mA} / \mathrm{cm}^{2}$, and the voltage was $<80 \mathrm{~V}$.

\section{Evaluations}

Measurements were performed at the baseline at the start of training as well as at the midpoint, and again at 6 weeks after the end of the exercise program, by a single blinded assessor.

Muscle Strength: Maximal isometric left wrist flexion and extension torques were obtained with the subject sitting in a CYBEX6000 (CSMI, MA, USA) apparatus with the left wrist positioned in the neutral position. Each subject was then asked to produce a maximal isometric wrist flexion and extension, and encouraged to monitor their own efforts on a video screen. The MVC was defined as the mean of three measurements at 5-min intervals.

Left grip strengths (GS) were measured with a hand-held Jamar dynamometer (Takei Kiki Kogyo Co., Ltd., Niigata, Japan) and defined as the mean of three measurements at 5-min intervals, using procedures recommended by the American Society of Hand Therapists [21-23].

Muscle mass: Cross sectional area (CSA)s in the forearm flexor and extensor were determined in conjunction with the torque evaluation sessions. The midpoint between the humeral lateral epicondyle and the radial styloid process was marke (Hitachi Medical Corp, Tokyo, Japan) with an indelible marker at the baseline evaluation and renewed as necessary throughout the experiment. A $5 \times 5 \times 5 \mathrm{~mm}$ magnetic resonance imaging (MRI) marker (Chugai Pharmaceutical Co., Ltd., Tokyo, Japan) was placed on the mark at the time of a CSA determination. Scout films were used to ensure that the scanning position did not change between sessions and images were collected as the subject lay with the upper extremity in a relaxed state. CSAs were measured by a blinded observer on the display of MRI scanner (Hitachi Medical Co., Tokyo, Japan).

Hand function: Two tests were used to assess different aspects of hand function. The standard version of the Purdue Pegboard (PEG) test was used to provide a global assessment of dexterity; Tiffin et al. $[24,25]$ describe it in detail in their the manual. The PEG test provides separate sub-tests of the $\mathrm{RH}$, left hand ( $\mathrm{LH})$, both hands $(\mathrm{BH})$, right plus left plus both hands (RLB), and assembly test (Assembly) [28]. We took the three-trial administration procedures of the PEG test, and used average sub-test scores for our estimate $[25,26]$. The finger tapping (Tapping) test was used to assess speed of finger movements [26], and involved tapping the same key on a notebook computer with the index finger as fast as possible for ten sec of testing time, while not moving the wrist. The score was the number of times tapped.

Subjects were questioned about the occurrence of adverse effects at each session. Goniometric active and passive ranges of motion (ROM) as well as complaints of muscle pain were evaluated at the beginning and end of each session.

\section{Data Analysis}

A linear mixed model [27] was employed to com- 
pare training effects on torques, GS, CSAs, PEG test scores and Tapping test scores between the three experimental groups. In the model, changes of measurements, (at the midpoint and endpoint of training) from the baseline were used as outcomes, physical characteristics (age, height and weight) and baseline scores were defined as co-variates, and random intercepts were included accounting for both heterogeneity of baseline measurements and serial correlation among repeated measurements. Comparison was made of the changes in measurements from the baseline within each group and between groups. Statistical analyses were performed with SAS software and $p$ values $\leq 0.05$ were considered to be statistically significant. Data are presented as means $\pm \mathrm{SD}$.

\section{RESULTS}

\section{Muscle Strength}

Wrist flexion torque (Fig. 1A): Maximal isometric wrist flexion torque in the HYB group increased from $12.8 \pm 4.1 \mathrm{Nm}$ at the pre-training to $15.0 \pm 4.0 \mathrm{Nm}$ at the end of training, however this increase was not significant. Maximal isometric wrist flexion torque did not change in the $\overline{\mathrm{ES}}$ (from 13.8 $\pm 5.0 \mathrm{Nm}$ at pretraining to $14.1 \pm 3.0 \mathrm{Nm}$ at the end of training) or $\mathrm{CN}$ groups (from 11.2 $\pm 4.1 \mathrm{Nm}$ at pre-training to $12.2 \pm 3.6$ $\mathrm{Nm}$ at the end of training). No significant differences were observed between the groups.

Wrist extension torque (Fig. 1B): Maximal isometric wrist extension torque in the HYB group significantly increased from $11.4 \pm 3.7 \mathrm{Nm}$ at pre-training to $14.0 \pm 3.1 \mathrm{Nm}$ at the end of training $(\mathrm{p}<0.01)$. The maximal isometric wrist extension torque did not change in the ES (from $10.5 \pm 3.6 \mathrm{Nm}$ at pre-training to $11.1 \pm 2.9 \mathrm{Nm}$ at the end of training) or $\mathrm{CN}$ groups (from 11.6 $\pm 3.2 \mathrm{Nm}$ at pre-training to $10.3 \pm 3.1 \mathrm{Nm}$ at the end of training). The increase of maximal isometric wrist extension torque was larger in the HYB group than in the $\mathrm{CN}$ group at the midpoint of the training (p $<0.01)$ and at the endpoint of the training $(\mathrm{p}<0.01)$.

$\boldsymbol{G S}$ : There were no significant changes in GS for any of the three groups (Table 1).

\section{Muscle mass}

CSA of flexor (Fig. 2A): Forearm flexor CSA in the HYB group increased significantly from $13.4 \pm 2.5$ $\mathrm{cm}^{2}$ at pre-training to $14.4 \pm 3.0 \mathrm{~cm}^{2}$ at the midpoint of training $(\mathrm{p}<0.05)$, and to $14.7 \pm 2.9 \mathrm{~cm}^{2}$ at the end of training $(\mathrm{p}<0.01)$. Forearm flexor CSA did not change in the $\mathrm{ES}$ or $\mathrm{CN}$ groups, $\left(13.4 \pm 2.5 \mathrm{~cm}^{2}\right.$ at pre-training

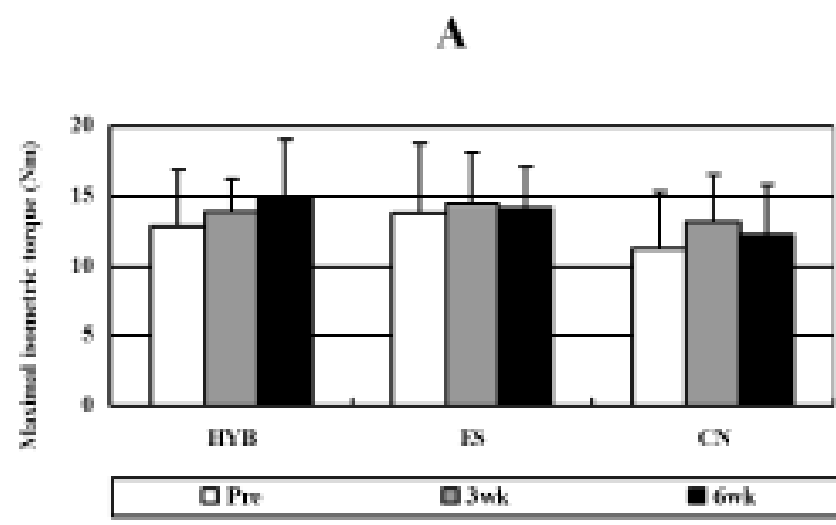

B

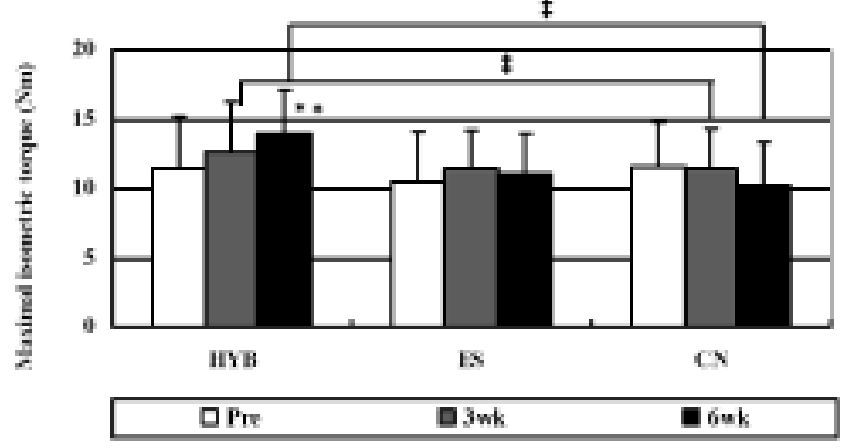

Fig. 1. Maximal isometric wrist flexion (A) and extension (B) torque production $(\mathrm{Nm})$ at pre-training baseline as well as at the 3-week midpoint of training, and its 6-week conclusion. (A) Wrist flexion torque production had not increased in a statistically significant manner at the end of the 6-week training program in both the HYB and CN groups. Wrist extension torque production increased in the HYB groups by the end of training $(\mathrm{p}<0.01)$. (B) Both at the midpoint of training and at the end of training, there were statistically significant differences between the HYB and $\mathrm{CN}$ groups $(\mathrm{p}<0.01)$. Values are means \pm SE. $*$ Significant differences from pre-training value, $\mathrm{p}<0.05 ; * * \mathrm{p}<0.01$. 'Significant differences between the groups, $\mathrm{p}<0.05$; $\stackrel{\mathrm{p}}{\mathrm{p}}<0.01$. HYB, hybrid exercise method; ES, electrical stimulation; CN, control; Pre, pre-training; 3wk, 3-week; 6wk, 6-week.

TABLE 1.

Results of grip strengths $(\mathrm{kg})$

\begin{tabular}{cccc}
\hline group & $\begin{array}{c}\text { Pre } \\
\text { mean }(\mathrm{SD})\end{array}$ & $\begin{array}{c}3 w \mathrm{wk} \\
\text { mean }(\mathrm{SD})\end{array}$ & $\begin{array}{c}\text { 6wk } \\
\text { mean }(\mathrm{SD})\end{array}$ \\
\hline HYB & $38.49(4.37)$ & $38.52(3.35)$ & $37.38(3.86)$ \\
ES & $41.07(6.21)$ & $40.89(6.80)$ & $40.72(6.93)$ \\
CN & $40.25(4.90)$ & $39.37(4.82)$ & $38.56(7.40)$ \\
\hline
\end{tabular}

Values are means (SD), HYB, hybrid exercise method; ES, electrical stimulation; $\mathrm{CN}$, control Pre, pre-training; 3wk, 3-week; 6wk, 6-week. 
A

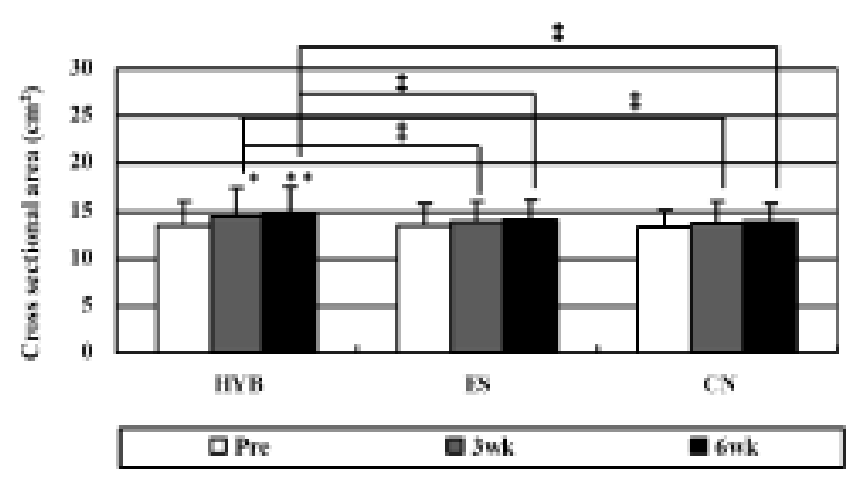

B

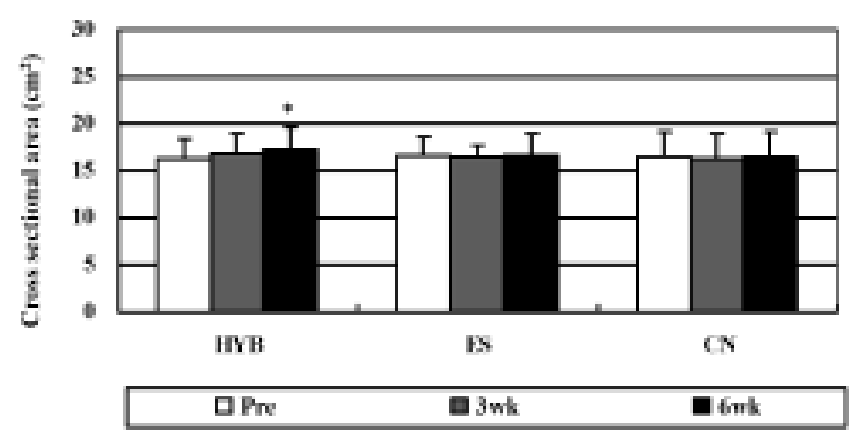

Fig. 2. Changes in the CSA $\left(\mathrm{cm}^{2}\right)$ of forearm flexor (A) and forearm extensor (B) at the pre-training baseline as well as at the 3 -week midpoint, and the 6-week conclusion of training. The forearm flexor CSA increased in the HYB groups by the end of training $(p<0.01)$. Both at the midpoint of training and at the end of training, there were statistically significant differences between the HYB and $\mathrm{CN}$ groups and between the HYB and ES groups, respectively $(\mathrm{p}<0.01)$. (A). Training effects on forearm extensor CSA were only evident in the HYB group statistically significant at the end of training $(\mathrm{p}<0.05)(B)$. Values are means \pm SE. $*$ Significant differences from pre-training value, $\mathrm{p}<0.05$; $* * \mathrm{p}<0.01$. 'Significant differences between the groups, $\mathrm{p}<0.05 ; \mathrm{p}<0.01$. HYB, hybrid exercise method; ES, electrical stimulation; $\mathrm{CN}$, control; Pre, pre-training; 3wk, 3-week; 6wk, 6-week.

to $14.1 \pm 2.2 \mathrm{~cm}^{2}$ at the end of training, and $13.3 \pm 1.7$ $\mathrm{cm}^{2}$ at pre-training to $13.9 \pm 1.9 \mathrm{~cm}^{2}$ at the end of training, respectively). The increase of the forearm flexor CSA in the HYB group was larger than that in the ES group or in the $\mathrm{CN}$ group at the midpoint of the training ( $<<0.01$, for each group) and at the endpoint of the training ( $\mathrm{p}<0.01$, for each group).

CSA of extensor (Fig. 2B): The forearm extensor CSA in the HYB group increased significantly from $16.27 \pm 2.2 \mathrm{~cm}^{2}$ at pre-training to $17.1 \pm 2.5 \mathrm{~cm}^{2}$ at the
TABLE 2.

Results of Purdue Pegboard test and finger tapping test

\begin{tabular}{ccrrr}
\hline group & test & $\begin{array}{c}\text { Pre } \\
\text { mean (SD) }\end{array}$ & $\begin{array}{c}\text { 3wk } \\
\text { mean (SD) }\end{array}$ & \multicolumn{1}{c}{$\begin{array}{r}\text { 6wk } \\
\text { mean (SD) }\end{array}$} \\
\hline HYB & RH & $17.7(1.16)$ & $18.7(1.34)$ & $18.3(1.64)$ \\
& LH & $16.7(1.77)$ & $17.1(1.85)$ & $17.5(1.51)$ \\
& BH & $13.8(1.18)$ & $14(1.40)$ & $14.9(1.14)$ \\
& RLB & $47.8(3.36)$ & $48.5(4.20)$ & $50.5(3.37)$ \\
& Assembly & $45.1(4.23)$ & $48.6(5.72)$ & $49.3(4.35)$ \\
& Tapping & $62(4.50)$ & $60.9(6.26)$ & $60(5.16)$ \\
\hline \multirow{2}{*}{ ES } & RH & $16.8(1.14)$ & $17.8(1.48)$ & $17.8(1.93)$ \\
& LH & $15.8(1.23)$ & $16.3(0.82)$ & $16.8(1.23)$ \\
& BH & $13.5(1.23)$ & $13.8(1.33)$ & $14.2(1.29)$ \\
& RLB & $45.6(2.72)$ & $47.4(4.81)$ & $48.2(3.65)$ \\
& Assembly & $43.9(3.84)$ & $45(5.45)$ & $46.3(7.03)$ \\
& Tapping & $57.9(4.99)$ & $55.3(5.27)$ & $52.3(2.80)$ \\
\hline \multirow{2}{*}{ CN } & RH & $17.4(1.58)$ & $18(2.00)$ & $18.5(1.72)$ \\
& LH & $16.1(1.10)$ & $16.3(1.83)$ & $17(1.05)$ \\
& BH & $13.4(0.84)$ & $13.6(1.71)$ & $14.9(1.60)$ \\
& RLB & $46.5(2.67)$ & $47(3.03)$ & $49.7(3.88)$ \\
& Assembly & $45.4(4.86)$ & $43.8(5.27)$ & $45.9(5.81)$ \\
& Tapping & $58.5(6.38)$ & $57.4(6.70)$ & $56.4(4.30)$ \\
\hline
\end{tabular}

Values are means (SD), HYB, hybrid exercise method; ES, electrical stimulation; CN, control; Assembly, assembly test; $\mathrm{RH}$, right hand; $\mathrm{LH}$. left hand; $\mathrm{BH}$, both hands; RLB, right plus left plus both hand; Pre, pre-training; 3wk, 3-week; 6wk, 6-week.

end of training $(\mathrm{p}<0.05)$. The forearm extensor CSA did not increase from pre-training to the end of training in the $\mathrm{ES}$ or $\mathrm{CN}$ group $\left(16.57 \pm 2.0 \mathrm{~cm}^{2}\right.$ to $16.62 \pm$ $2.3 \mathrm{~cm}^{2}$, and $16.4 \pm 2.6 \mathrm{~cm}^{2}$ to $16.4 \pm 2.8 \mathrm{~cm}^{2}$, respectively). There were no significant differences between the groups.

\section{Hand function}

PEG and Tapping test: The scores of PEG test (RH, LH, BH, RLB, and Assembly) and Tapping test showed no significant changes within or among any of the three groups (Table 2).

The subjects tolerated training well and attended all training and evaluation sessions. There were no significant adverse events, and ROM was preserved in all groups. Four subjects in the HYB group complained of mild delayed onset muscle soreness during the first week of training, but recovered by the following week.

\section{DISCUSSION}

This study showed that HYB significantly increased wrist isometric extension torque and wrist muscle mass in both the flexor and extensor without having any 
negative influence on hand functions. Although our sample size and the muscles studies were limited, these findings deserve further discussion.

It is well known that immobilization or disuse cause muscle atrophy, that is to say muscle mass, strength and endurance decrease [28-30]. Various training approaches are used in rehabilitation medicine to recover from immobilization or disuse. NMES is used widely to lessen immobilization-associated muscle weakness, to strengthen muscles, and to improve function in people with neuromuscular disabilities $[1,19,28,32]$. For example, the muscle strength of healthy subjects can be increased by $10 \%$ to $20 \%$ with 3 to 6 weeks of highintensity stimulation [4,5]. Also, the usefulness of an electrically stimulated eccentric contraction was reported $[33,34]$. However, NMES has some limitations. NMES activates slow-twitch muscle poorly because Henneman's size principle is not seen in electrical stimulation [6]. Moreover, it is hard to activate the deep muscles using NMES with surface electrodes, and high electrical stimulation intensity causes discomfort. Therefore, combining NMES and VC can help overcome the limitations that occur when each is used in isolation, and is more effective for improving muscle properties $[8,9]$. HYB was developed as a new type of combined technique. It was reported effective in improving muscle strength and mass in healthy subjects [11-13]. This HYB obviates the weak point of NSEM because the use of both VC and ES makes it a more physiologic approach [11]. It is thought that the deep muscles that are difficult to stimulate by means of surface electrical stimulation are trained adequately by volitional contraction in HYB [11-14]. Also, HYB involves a combination of eccentric contractions and electric stimulation. Segar and Thorstensson [33] compared the effects of electrically stimulated eccentric, isometric, and concentric contractions of the quadriceps, and found that under similar stimulating conditions, eccentric contractions generated torques about $15 \%$ to $25 \%$ greater than those produced with isometric contractions and about $30 \%$ to $50 \%$ greater than the torques resulting from concentric contractions. Therefore, it has been considered that HYB increases muscle strength satisfactorily even at relatively low stimulation intensities. Indeed, Takano et al. [14] showed that HYB improved thigh muscle strength and hypertrophy in elderly people with a relatively low electrical stimulation intensity (equivalent to 20-25 repetition maximal). These advantages show that HYB is effective even for sites where high stimulation intensity is problematic, for example the forearm. The present study showed that HYB was effective in the forearm in healthy subjects, except with regard to GS and wrist flexion torque. On the other hand, NMES alone had no effect in the forearm at the same electrical stimulation intensity and frequency. Generally, the training effects of NMES are correlated to the stimulation intensity [8]. Because we made the electrical stimulation intensity weak enough to prevent discomfort, NMES would be ineffectiveness. The results of this study demonstrate that training became more effective when voluntary contractions were combined with NMES, even at a low electrical stimulation intensity (insufficient in NMES alone). In other words, HYB appears more efficient than NMES or VC alone.

NMES and VC induce different acute physiological effects on the neuromuscular system [9]. However, there it is not yet known how the combined application of NMES and VC influences neuromuscular systems. Therefore, HYB might induce an adverse influence on the neuromuscular system. Development of a training approach that can be used on all parts of the body is highly desirable because muscle weakness can occur anywhere in the body. Rehabilitation of the upper extremity is necessary not only for disuse but also for paralysis and rheumatoid arthritis. It has been reported that NMES is effective in paralysis $[17,32]$ and rheumatoid arthritis [31]. In rehabilitation of the upper extremities (especially the forearm, hand, and finger), not only the effect of muscle strength but also the effect of hand function is very important $[15,16]$. Therefore the possible adverse influence on the specific coordination of complex movements needs to be considered in order to use HYB safely. Because NMES only stimulates the muscle on which the electrodes are placed, synergic and stabilizer muscles were not activated by NMES [9]. Paillard reported that NMES did not facilitate intermuscular coordination [9]. Indeed Vaz et al. [17] showed that NMES on wrist with hemiplegic cerebral palsy increased wrist strength without changing hand function. On the other hand, NMES of Ia fibre in the extensor carpi radialis muscle is thought to induce reciprocal Ia-inhibition in the hand and finger flexors in stroke patients [18]. Also, Maffiuletti et al. [35] reported that NMES in plantar flexor muscles did not affect alpha motoneuron excitability and/or presynaptic inhibition by examining maximal soleus and gastrocnemii $\mathrm{H}$ - and T-reflex. Voluntary training-induced modifications in H-reflex amplitude are usually ascribed to changes in alpha motoneuron excitability and/or changes in presynaptic inhibition of Ia afferents. However, these effects are not well understood in the combined technique. In the only report about the effect on H-reflex of HYB by Yamaguchi et al. [36], 
they showed that the rate of change of the maximum soleus H-reflex to maximum soleus motor response in HYB was significantly higher than in conventional resistance training. They suggested that HYB was similar to co-contraction, and effects supraspinal and center mechanisms differently from conventional resistance training. If the specific coordination of complex movements in the upper extremity is disturbed by the combined technique, hand function would decline. In this study, we used PEG test and Tapping test to evaluate hand function, and results were not effected by HYB. This seems to show that HYB does not adversely affect the specific coordination of complex movements. Further research is necessary to reveal the effects on the neuromuscular system of the combined application of NMES and VC.

Isometric wrist flexion torque was not increased significantly in this study, because the wrist position might influence the measurement value [37]. Vaz et al. [17], in a study of NMES training on wrist muscles of children with hemiplegic cerebral palsy, reported that the isometric strength of flexors did not increase at the neutral position despite an increase of $30^{\circ}$ in wrist extension, possibly reflecting shifts in the length-tension relationships due to functioning in altered muscle lengths. In the present study torque measurements were carried out in the neutral position, however there were no significant effects in either wrist isometric flexor strength or GS. Therefore the HYB program which was used in the current study might not be appropriate. The potential strength gain benefits are generally correlated to the intensity of the stimulation and the frequency of the training sessions [8]. It is possible that exercised in higher intensity the results might have varied. In HYB, the motion of a volitionally contracting agonist muscle against the force generated by its electrically stimulated antagonist shows an effect in the deep muscles where the electrical stimulation cannot reach. Influence on the deep muscles including the flexor digitorum profundus muscle which was important for GS might be ineffective in this study. Furthermore, because of the wrist flex action of their muscles, the increase of maximal isometric wrist flexion torque might lack statistical power even if the forearm flexor was significantly enlarged. Electrical stimulation intensity and the training frequency that we used in this study were set based on the maximum comfortable intensity we used in improving muscle strength and mass in the upper arm in a previous study [13]. However, this stimulation intensity appeared inadequate to increase muscle strength of grip and wrist flexor. Because the maximum comfortable intensity differs by stimulus site or subjects' tolerance, it may be desirable to set electrical stimulation intensity based on the repetition maximum as described by Takano et al. [14]. Voluntary wrist flexion exercise load can be increased by regulating electrical stimulation intensity of the carpal extensor. Improving the effectiveness of HYB is a problem for future study. In any case, HYB was effective in strengthening the wrist extensor without having negative influences on hand function (as a parameter of the neuromuscular system). It will be necessary to evaluate influences on different parts of the neuromuscular system with various HYB programs.

The current study was designed to assess the possible adverse effects of HYB on the specific coordination of complex movements, and it is the first study utilizing HYB for the forearm. To achieve more positive effects for the wrist, hand, and finger forces, it may be necessary to alter the present exercise program. Further research is necessary to refine HYB techniques and more clearly demonstrate the potential benefits for rehabilitation medicine.

\section{CONCLUSIONS}

HYB was capable of increasing muscle mass and strength without having any adverse influence on hand functions. This technique may be an effective and safe exercise technique for rehabilitation medicine.

ACKNOWLEDGMENTS: This study was supported in part by the Japanese Society for the Promotion of Science Grant-inAid for scientific research (14350138).

\section{REFERENCES}

1. Kagaya H, Shimada Y, and Ebata K. Restoration and analysis of standing-up in complete paraplegia utilizing functional electrical stimulation. Arch Phys Med Rehabil 1995; 76:876-881.

2. Delitto A, Rose SJ, McKowen JM, Lehman RC, Thomas JA et al. Electrical stimulation versus voluntary exercise in strengthening thigh musculature after anterior cruciate ligament surgery. Phys Ther 1988; 68:660-663.

3. Snyder-Mackler L, Delitto A, Bailey SL, and Stralka SW. Strength of the quadriceps femoris muscle and functional recovery after reconstruction of the anterior cruciate ligament. A prospective, randomized clinical trial of electrical stimulation. J Bone Joint Surg Am 1995; 77:1166-1173.

4. Balogum JA, Onilari OO, Akeju OA, and Marzouk DK. High voltage effects of pulse frequency. Arch Phys Med Rehabil 1993; 74:910-916.

5. Stein RB, Kimoto M, and Bobet J. Biomechanics of human quadriceps muscle during electrical stimulation. J Bionmech 1999; 32:347-353.

6. Henneman E, Somjen G, and Carpenter DO. Functional 
significance of cell size in spinal motoneurons. J Neurophysiol 1965; 28:560-580.

7. Gregory CM, and Bickel CS. Recruitment patterns in human skeletal muscle during electrical stimulation. Phys Ther 2005; 85:358-364.

8. Dehail P, Duclos C, and Barat M. Electrical stimulation and muscle strengthening. Ann Readapt Med Phys 2008; 51:441-451.

9. Paillard T. Combined application of neuromuscular electrical stimulation and voluntary muscular contractions. Sports Med 2008; 38:161-177.

10. Cabric M, and Appell HJ. Effect of electrical stimulation of high and low frequency on maximum isometric force and some morphological characteristics in men. Int J Sports Med 1987; 8:256-260.

11. Yanagi T, Shiba N, Maeda T, Iwasa K, Umezu Y et al. Agonist contractions against electrically stimulated antagonists. Arch Phys Med Rehabil 2003; 84:843-848.

12. Iwasaki T, Shiba N, Matsuse H, Nago T, Umezu Y et al. Improvement in Knee Extension Strength through Training by Means of Combined Electrical Stimulation and Voluntary Muscle Contraction. Tohoku J Exp Med 2006; 209:33-40.

13. Matsuse H, Shiba N, Umezu Y, Nago T, Tagawa Y et al. Muscle training by means of combined electrical stimulation and volitional contraction. Aviat Space Environ Med 2006; 77:581-585.

14. Takano Y, Haneda Y, Maeda T, Sakai Y, Matsuse H et al. Increasing muscle strength and mass of thigh in elderly people with the hybrid-training method of electrical stimulation and volitional contraction. Tohoku J Exp Med 2010; 221:77-85.

15. Brorsson S, Hilliges M, Sollerman C, and Nilsdotter A. A six-week hand exercise programme improves strength and hand function in patients with rheumatoid arthritis. J Rehabil Med 2009; 41:338-342.

16. Olafsdottir HB, Zatsiorsky VM, and Latash ML. The effects of strength training on finger strength and hand dexterity in healthy elderly individuals. J Appl Physiol 2008; 105:1166-1178.

17. Vaz DV, Mancini MC, da Fonseca ST, Arantes NF, Pinto TB et al. Effects of strength training aided by electrical stimulation on wrist muscle characteristics and hand function of children with hemiplegic cerebral palsy. Phys Occup Ther Pediatr 2008; 28:309-325.

18. Hultborn H, Illert M, and Santini M. Convergence on interneurones mediating the reciprocal Ia inhibition of motoneurones. I. Disynaptic Ia inhibition of Ia inhibitory interneurones. Acta Physiol Scand. 1976; 96:193-201.

19. Oldfield RC. The assessment and analysis of handedness: the Edinburgh inventory. Neuropsychologia 1971; 9:97-113.

20. Ward AR, and Shkuratova N. Russian electrical stimulation: the early experiments. Phys Ther 2002; 82:1019-1030.

21. Fess EE. Clinical assessment recommendation. In: American Society of Hand Therapists Monograph (2nd ed.), Indianapolis, 1991.

22. Fess EE. The effect of Jamar dynamometer handle position and test protocol on normal grip strength. J Hand Surg 1982; 7A:308-309.

23. Hinson M, Woodard J, and Gench B. Reliability of the Jamar digital dynamometer model 2A. Occup Ther J Res 1990; 10:108-110.

24. Buddenberg LA, and Davis C. Test-retest reliability of the Purdue Pegboard Test. Am J Occup Ther. 2000; 54:558-558.

25. Tiffin J, and Asher EJ. The Purdue Pegboard; norms and studies of reliability and validity. J Appl Psychol 1948; 32:234-247.

26. Kee D, Morris K, Bathurst K, and Hellige JB. Lateralized interference in finger tapping: comparisons of rate and variability measures under speed and consistency tapping instructions. Brain Cogn 1986; 5:268-279.

27. Tokizane T. Studies on the paradoxical phase of sleep in the cat. In: Progress in Brain Research, ed. Tokizane T and Schade JP, Elsevier, Amsterdam, Vol 21B, pp 230-268, 1965.

28. Appell HJ. Muscular atrophy following immobilisation. A review. Sports Med 1990; 10:42-58.

29. Fiatarone MA, O'Neill EF, Ryan ND, Clements KM, Solares GR et al. Exercise training and nutritional supplementation for physical frailty in very elderly people. N Engl J Med 1994; 330:1769-1775.

30. Kozlovskaya IB, and Grigoriev AI. Russian system of countermeasures on board of the International Space Station (ISS): the first results. Acta Astronautica 2004; 55:233-237.

31. Brosseau LU, Pelland LU, Casimiro LY, Robinson VI, Tugwell PE et al. Electrical stimulation for the treatment of rheumatoid arthritis. Cochrane Database Syst Rev 2002; CD003687.

32. Hummelsheim H, Maier-Loth ML, and Eickhof C. The functional value of electrical muscle stimulation for the rehabilitation of the hand in stroke patients. Scand J Rehabil Med 1997; 29:3-10.

33. Segar JY, and Thorstensson A. Electrically evoked eccentric and concentric torque-velocity relationships in human knee extensor muscles. Acta Physiol Scand 2000; 169:6369.

34. Westing SH, Segar JY, and Thorstensson A. Effects of electrical stimulation on eccentric and concentric torquevelocity relationships during knee extension in man. Acta Physiol Scand 1990;140:17-22.

35. Maffiuletti NA, Pensini M, Scaglioni G, Ferri A, Ballay Y et al. Effect of electromyostimulation training on soleus and gastrocnemii $\mathrm{H}$ - and T-reflex properties. Eur J Appl Physiol 2003; 90:601-607.

36. Yamaguchi $\mathrm{T}$, Tanabe $\mathrm{S}$, Watanabe $\mathrm{T}$, and Muraoka Y. Effect of voluntary contraction with electrical stimulation to antagonist muscle on agonist H-reflex. Electromygr Clin Neurophysiol 2007; 47:251-255.

37. Bialocerkowski A, and Grimmer KA. Measurement of isometric wrist muscle strength--a systematic review of starting position and test protocol. Clin Rehabil 2003; 17:693702 . 